\title{
Uso del condón y conocimientos sobre prevención del VIH entre los inyectadores de drogas de dos ciudades colombianas
}

\section{Use of the condom and knowledge on HIV prevention among the injector's drugs in two Colombian cities}

\author{
Dedsy Berbesí-Fernández, Liliana Montoya-Vélez, Ángela Segura-Cardona \\ Facultad de Medicina. Universidad CES, Medellín, Colombia
}

\section{Resumen}

Se pretende describir la utilización del condón en usuarios de droga por vía intravenosa e identificar los conocimientos sobre prevención del VIH que puedan orientar intervenciones. Se realizó un estudio cross-sectional, utilizando el muestreo guiado por el entrevistado (RDS en sus siglas en inglés). La muestra estuvo compuesta por 540 participantes, mayores de 18 años, de dos ciudades colombianas. La encuesta incluía asesoramiento para la prueba de VIH y para la prevención de enfermedades de transmisión sexual. En el análisis multivariado se calculó un modelo de regresión logística multinomial (explicativo de utilización del condón), con los OR crudos y ajustados. La proporción de usuarios de droga por vía intravenosa que utiliza siempre condón en sus relaciones sexuales con sus parejas fue de $21,1 \%$. Se encontró que al ajustar el riesgo de no utilización del condón por los diez conocimientos inadecuados sobre prevención del VIH, los factores que aumentan el riesgo son el compartir el equipo de inyección (ORaj=3,19 [1,29-7,88]), el tener sexo de manera insegura (ORaj=3,74 [1,21-11,58]) y el no aumentar el uso del condón a pesar de tener los conocimientos sobre VIH (ORaj=33,59 [9,19-122,86]). La baja utilización del condón en los usuarios de droga por vía intravenosa está asociada con los conocimientos inadecuados sobre la prevención del VIH.

Palabras clave: VIH, conducta sexual, heroína, preservativos, Colombia.

\begin{abstract}
This research focuses on the use of the condom among injector drug users and the identification of their knowledge on HIV prevention that can inform interventions. A cross- sectional study was conducted, using respondent driven sampling (RDS); the sample was composed of 540 participants, of 18 years of age or older. The survey included counseling for HIV testing and the prevention of sexually transmitted diseases. In the multivariate analysis estimated a logistic regression model (explaining how to use the condom) multinomial with raw and adjusted OR. The proportion of drug-injector users who always used condoms during sexual intercourse with their partners was $21.1 \%$. It was found that by adjusting the risk of not using a condom by ten inadequate assumptions on HIV prevention, factors that increase the risk are sharing the injection equipment $($ ORaj $=3.19[1.29-$ 7.88]), having unsafe sex practices (ORaj = 3. 74 [1. 21-11. 58]) and not increasing the use of condom despite having knowledge of HIV $($ ORaj $=33.59$ [9. 19-122. 86] $)$. The low use of condoms among intravenous drug users is associated with inadequate knowledge about HIV prevention.
\end{abstract}

Key words: HIV, sexual behavior, heroin, preservatives, Colombia. 


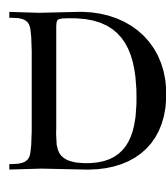

e acuerdo a la Declaración Política sobre el $\mathrm{VIH} /$ Sida de las Naciones Unidas, la infección del VIH sigue siendo una pandemia sin precedentes que produce un sufrimiento inmenso a los países de todo el mundo, con más de 30 millones de personas muertas a causa del SIDA, un estimado de 33 millones de personas viviendo con el VIH, 7000 infecciones nuevas, y menos de la mitad de las personas que viven con el VIH conocen su diagnóstico (Naciones Unidas, 2011). En casi todas las regiones del mundo, fuera del África subsahariana, el VIH afecta en mayor proporción a hombres que tienen sexo con hombres y a personas usuarias de drogas inyectables (ONUSIDA, 2012).

El uso de drogas intravenosas ha sido identificado como un factor de riesgo desde el inicio de la epidemia del VIH-Sida; se calcula que, en todo el mundo, hay unos 3 millones de usuarios de drogas inyectables que viven con el VIH, mientras que 13 millones más corren el riesgo de infectarse (Mathers et al., 2010). Los usuarios de drogas inyectables (UDI) por sus prácticas sexuales, tienen mayor riesgo de adquirir enfermedades de transmisión sexual y en especial VIH (NIDA, 2001). Las mujeres también presentan mayor tendencia a encontrarse inmersas en conductas sexuales riesgosas como intercambiar sexo por dinero o drogas, tener múltiples parejas sexuales, compartir agujas durante la relación sexual o tener sexo no protegido con una pareja UDI (Berbesi, Montoya y Segura, 2012).

Los UDI son el foco de la atención sanitaria en intervenciones dirigidas a reducir el comportamiento de riesgo asociados con el abuso de drogas (por ejemplo, el intercambio de agujas). Sin embargo, parece que los UDI son más fáciles de convencer para dejar de compartir agujas que para modificar su comportamiento sexual (Ball, Rana y Dehne, 1998). Actualmente, en muchos países los consumidores de drogas están reduciendo los daños directamente asociados al consumo endovenoso. Sin embargo, simultáneamente a lo anterior, la transmisión del VIH por vía sexual empezó a incrementarse nuevamente, convirtiéndose, en la principal forma de transmisión de VIH en los UDI (March, Oviedo-Joekes y Romero, 2007; Mendez Diz, Schwarz y Camarotti, 2009).

La vía de propagación del VIH desde la comunidad de UDIs a la población general es la vía sexual (Carnicer-Pont, Vives y Casabona i Barbarà, 2011). Estos usuarios suelen ser personas jóvenes y sexualmente activas. Sus parejas sexuales pueden ser, tanto personas que se inyectan como personas que no se inyectan drogas. Por el momento, el condón constituye el medio de mayor eficacia probada en la prevención de la transmisión sexual del VIH (Chiao, Morisky, Ksobiech y Malow, 2009). Por esta razón, el aumento del uso del preservativo se ha convertido en una intervención clave destinada a proteger a las personas contra la infección de VIH tanto para los grupos más vulnerables, así como la población general (Bonell y Imrie, 2001).
Por las características de la epidemia de VIH/Sida en Colombia de naturaleza concentrada es importante avanzar en la priorización y focalización de acciones en grupos altamente vulnerables, con acciones de vigilancia de segunda generación (Organizacion Mundial de la Salud y ONUSIDA, 2000) y la identificación de comportamientos de riesgo que permita conocer el aporte que podrían estar haciendo estos grupos al perfil actual y futuro de la epidemia de VIH (Ministerio de la Proteccion Social. Republica de Colombia, 2008).

En Colombia no se habían realizado estudios sobre la utilización del condón en usuarios de drogas por vía intravenosa. El objetivo de este trabajo es describir la utilización del condón por usuarios de droga por vía intravenosa e identificar los conocimientos sobre prevención del VIH que puedan ser factores asociados a esta utilización y que puedan ser útiles en la formulación de estrategias de prevención de la transmisión del VIH.

\section{Método}

Se realizó un estudio cross-sectional, de usuarios activos de drogas inyectables, en dos ciudades de Colombia en el segundo semestre de 2011, utilizando el muestreo guiado por el participante.

Debido a la características de la población estudiada, no fue posible contar con un marco muestral conocido que permitiera diseñar una muestra probabilística convencional, por lo que se optó por un muestreo guiado por el entrevistado, método que ha comprobado su utilidad en poblaciones de difícil acceso como son los usuarios de drogas inyectables; este muestreo incorpora teorías de redes sociales para obtener una muestra que se aproxime a la población estudiada (Abdul-Quader, Heckathorn, Sabin y Saidel, 2006).

El reclutamiento se inició con un grupo de "semillas" o participantes no aleatorios de la población objetivo, su selección se hizo a través de informantes claves, los cuales reclutaron a otros compañeros que cumplieran los criterios de inclusión: tener más de 18 años de edad y ser consumidor de drogas por vía inyectable.

Se determinó una cantidad inicial de tres semillas para cada ciudad. Desde estas semillas se iniciaron las cadenas de reclutamiento, lográndose una muestra de 540 participantes (240 en Medellín y 300 en Pereira). A estas personas se les entregó dos tipos de incentivos, un incentivo primario o por participar en el estudio y otro secundario, vinculado al reclutamiento exitoso de tres nuevos participantes (los valores variaron entre aproximadamente US\$ 4 y US\$ 5, respectivamente). El tamaño de la muestra objetivo se alcanzó en un plazo de ocho semanas. Los requerimientos éticos fueron aprobados por un Comité de Ética en investigación. 
Para medir conocimientos frente al VIH/Sida se utilizó la encuesta de comportamiento en consumidores de drogas con alto riesgo (CODAR), se tomaron 10 reactivos sobre los conocimientos sobre VIH, el análisis factorial confirmó la estructura de la escala y un análisis de confiabilidad con un Alfa de Cronbach 0.74 para el instrumento.

Se realizó análisis multivariado calculando un modelo de regresión logística multinomial (explicativo de la frecuencia en la utilización del condón) calculando los OR crudos y después los OR ajustados con el método de Main effect, con sus intervalos de confianza del $95 \%$ y pruebas de significación estadística menores del 5\%; La pregunta que se realizó fue: ¿en los últimos seis meses con qué frecuencia utilizaron condones en los contactos sexuales?; la categoría de referencia fue la utilización del condón siempre; las categorías que se compararon fueron nunca y a veces.

\section{Resultados}

\section{Características socio demográficas, consumo de drogas y comportamiento sexual}

Los usuarios de drogas inyectables residían en dos ciudades de Colombia; con gran predominio de hombres jóvenes con estudios de secundaria; y un promedio de casi cuatro años de estar utilizando drogas inyectables, tenían como fuente principal de ingresos la venta ambulante y un $11,25 \%$ de los UDI obtenían sus ingresos de actividades ilegales; asi mismo un $42 \%$ reportó compartir agujas o parte del equipo de inyección durante los últimos seis meses; y 6 de cada 10 se inyectaban diariamente.

El 76,1\% de los UDI informaron haber tenido contacto sexual en los últimos seis meses; el 9,8\% eran hombres que tenían sexo con hombres (HSH) y un 34,2\% alguna vez en la vida ha tenido una sobredosis.

\section{Conocimientos de formas de prevención del VIH}

El $80 \%$ de los UDI tienen conocimientos con respecto a la transmisión del VIH por compartir agujas, pero esta proporción disminuye al $46 \%$ al considerar que otras partes del equipo de inyección puedan ser un vehículo para la transmisión del virus (tabla 1).

Con respecto a los conocimientos sobre la transmisión del VIH por las relaciones sexuales, el $65 \%$ de los UDI reconocen que el tener relaciones sexuales puede ser un factor de riesgo para el VIH y un $76 \%$ considera que aumenta el riesgo si se tienen relaciones sexuales sin protección (tabla 1).

Tabla 1

Conocimientos sobre prevención de VIH asociados a la frecuencia en la utilización del condón en UDI. 2010

\begin{tabular}{|c|c|c|c|c|c|c|c|c|c|c|c|c|}
\hline \multirow{3}{*}{\multicolumn{2}{|c|}{ Conocimientos y prácticas en VIH }} & \multicolumn{6}{|c|}{ Uso del condón nunca } & \multicolumn{5}{|c|}{ Uso del condón a veces } \\
\hline & & \multirow{2}{*}{$\mathrm{n}$} & \multirow{2}{*}{ OR c } & \multirow{2}{*}{ OR aj } & \multicolumn{2}{|c|}{ IC 95\% } & \multirow{2}{*}{$\begin{array}{l}\text { Valor } \\
\text { de p }\end{array}$} & \multirow{2}{*}{ OR c } & \multirow{2}{*}{ OR aj } & \multicolumn{2}{|c|}{ IC 95\% } & \multirow{2}{*}{$\begin{array}{l}\text { Valor } \\
\text { de } p\end{array}$} \\
\hline & & & & & LC Inf & LC sup & & & & LC Inf & LC sup & \\
\hline \multirow{2}{*}{$\begin{array}{l}\text { Compartiendo agujas } \\
\text { y/o jeringas }\end{array}$} & $\mathrm{Si}$ & 260 & 1,3 & 0,8 & 0,2 & 2,6 & 0,8 & 1,8 & 1,3 & 0,4 & 4,0 & 0,5 \\
\hline & No & 63 & 1,0 & 1,0 & - & - & - & 1,0 & 1,0 & - & - & - \\
\hline \multirow{2}{*}{$\begin{array}{l}\text { Compartiendo otro } \\
\text { equipo de inyección }\end{array}$} & $\mathrm{Si}$ & 115 & 1,7 & 0,8 & 0,2 & 3,03 & 0,7 & 1,3 & 0,7 & 0,2 & 2,56 & 0,6 \\
\hline & No & 134 & 1,0 & 1,0 & - & - & - & 1,0 & 1,0 & - & - & - \\
\hline \multirow{2}{*}{$\begin{array}{l}\text { Teniendo relaciones } \\
\text { sexuales }\end{array}$} & $\mathrm{Si}$ & 209 & 2,1 & 1,8 & 0,5 & 5,9 & 0,3 & 1,9 & 0,7 & 0,2 & 2,49 & 0,66 \\
\hline & No & 113 & 1,0 & 1,0 & - & - & - & 1,0 & 1,0 & - & - & - \\
\hline \multirow{2}{*}{$\begin{array}{l}\text { Teniendo sexo inseguro } \\
\text { o no protegido }\end{array}$} & $\mathrm{Si}$ & 207 & 2,2 & 3,7 & 1,2 & 11,5 & $0,0^{*}$ & 1,1 & 1,5 & 0,5 & 4,38 & 0,40 \\
\hline & No & 65 & 1,0 & 1,0 & - & - & - & 1,0 & 1,0 & - & - & - \\
\hline \multirow{2}{*}{$\begin{array}{l}\text { Teniendo contacto con } \\
\text { sangre infectada }\end{array}$} & $\mathrm{Si}$ & 169 & 2,0 & 2,6 & 0,8 & 8,6 & 0,1 & 1,9 & 1,4 & 0,4 & 4,49 & 0,51 \\
\hline & No & 123 & 1,0 & 1,0 & - & - & - & 1,0 & 1,0 & - & - & - \\
\hline \multirow{2}{*}{$\begin{array}{l}\text { Por transfusiones de } \\
\text { sangre/ productos } \\
\text { sanguíneos }\end{array}$} & $\mathrm{Si}$ & 101 & 1,9 & 1,9 & 0,5 & 7,0 & 0,3 & 2,5 & 1,8 & 0,5 & 5,99 & 0,30 \\
\hline & No & 130 & 1,0 & 1,0 & - & - & - & 1,0 & 1,0 & - & - & - \\
\hline \multirow{2}{*}{$\begin{array}{l}\text { Perinatalmente, de la } \\
\text { madre al hijo }\end{array}$} & $\mathrm{Si}$ & 39 & 3,7 & 1,1 & 0,1 & 10,4 & 0,8 & 4,7 & 1,5 & 0,1 & 15,07 & 0,68 \\
\hline & No & 168 & 1,0 & 1,0 & - & - & - & 1,0 & 1,0 & - & - & - \\
\hline \multirow{2}{*}{$\begin{array}{l}\text { Aumento el uso del } \\
\text { condón }\end{array}$} & No & 191 & 14,0 & 33,5 & 9,1 & 122,8 & $0,0 *$ & 3,2 & 5,1 & 1,4 & 18,97 & $0,01^{*}$ \\
\hline & $\mathrm{Si}$ & 77 & 1,0 & 1,0 & - & - & - & 1,0 & 1,0 & - & - & - \\
\hline \multirow{2}{*}{$\begin{array}{l}\text { Redujo la inyección de } \\
\text { drogas }\end{array}$} & No & 164 & 5,6 & 60,2 & 11,0 & 310,2 & $0,0^{*}$ & 7,6 & 48,9 & 48,9 & 48,96 & $0,00^{*}$ \\
\hline & $\mathrm{Si}$ & 20 & 1,0 & 1,0 & - & - & - & 1,0 & 1,0 & - & - & - \\
\hline \multirow{2}{*}{$\begin{array}{l}\text { Dejo de compartir } \\
\text { equipo/mezclas de } \\
\text { drogas }\end{array}$} & No & 120 & 0,3 & 0,3 & 0,1 & 0,8 & $0,0^{*}$ & 0,6 & 0,4 & 0,1 & 1,03 & 0,06 \\
\hline & $\mathrm{Si}$ & 112 & 1,0 & 1,0 & - & - & - & 1,0 & 1,0 & - & - & - \\
\hline
\end{tabular}


Se observó un bajo nivel de conocimientos en las preguntas relacionadas con fluidos de sangre: el $58 \%$ de los usuarios de drogas inyectables reconoce que el contacto con sangre infectada es una forma de transmisión del VIH y un $44 \%$ que las transfusiones de sangre también son un riesgo de la infección; solo un $19 \%$ de los usuarios de drogas inyectables reconoce que se puede transmitir de manera vertical el VIH de una madre infectada al hijo (tabla 1).

A la pregunta, si ha hecho algo para evitar infectarse desde que escuchó hablar de VIH, el 28,7\% refiere haber aumentado el uso del condón, el 10,9\% informó haber reducido la inyección de drogas y un 48,3\% refirió haber dejado de compartir el equipo de inyección; de esta manera se identifica una mayor percepción del riesgo de transmisión del VIH en actividades propias de la inyección que en las relaciones sexuales, y estas últimas son las que han aportado más en la transmisión del VIH en Colombia (tabla 1).

\section{Conocimientos sobre VIH y uso del condón}

Se ha reconocido que el tener conocimientos adecuados no siempre lleva a prácticas seguras para la prevención de la transmisión del VIH (ONUSIDA, 2012), las personas que informaron tener sexo inseguro tienen tres veces el riesgo en; este riesgo aumenta al ajustar por los demás conocimientos consultados (ORaj: 3,74, IC 95\%: 1,21 11,58) (Tabla 1).

Las personas que informaron no haber reducido la inyección de drogas, tenían mayor riesgo de no utilizar el condón, este factor se potencializaba al controlar por las demás variables consultadas de conocimientos de VIH (ORaj: 60, 25, IC 95\%: 11,02-310,23) (Tabla 1).

\section{Discusión}

La conducta sexual individual y el uso de condones son determinantes claves del estatus de riesgo de contraer infecciones de transmisión sexual y cuando esta información se agrega a nivel de la población, permite describir el contexto en el cual se deben diseñar e implementar los programas preventivos de VIH/Sida e ITS (USAID, 2010). Esta investigación es una aproximación a los conocimientos sobre VIH y la utilización del condón que permiten orientar las acciones educativas que deben reforzarse, y estrategias que permitan su aplicación en contextos difíciles, como en los que se encuentran los usuarios de drogas inyectables.

En Colombia, los UDI no se encuentran priorizados como una población en riesgo de transmisión del VIH, esta investigación identificó un número significativo de usuarios de drogas inyectables que tienen bajos conocimientos sobre prevención del VIH y un uso indebido de preservativos, factores de riesgo conocidos para el VIH. Desde hace varias décadas, en otros países, estos factores ya han sido ampliamente documentado y discutido (Bravo, Royuela, Barrio, Rodríguez-Arenas, y de la Fuente, 2004) pero para
Colombia esta investigación constituye un primer paso en la búsqueda de estrategias para prevenir la propagación adicional de la infección.

La población de usuarios de drogas inyectables fue de predominio masculino, similar a lo existente en otros estudios realizados 1983; Shisana, Rice, Zungu, \& Zuma, 2010 (Cobos Calleja, Casanueva Gutiérrez y Jove González, 2003; Hagan et al., 2007) pero se ha reconocido a las mujeres más expuestas a un comportamiento sexual de riesgo, específicamente respecto al rechazo por el uso del condón y quienes se ven desfavorecidas en la toma de decisiones y en la utilización de medidas de prevención (Uribe, Amador, Zacarias y Virrareal, 2012).

En Paraguay, en el estudio nacional de salud sexual y reproductiva, se identificó que los grupos de mujeres casadas o unidas, las de menor escolarización, así como las de los estratos socioeconómicos medio y bajo, tuvieron menos probabilidad de reportar uso de condón (Organización Mundial de la Salud, 2010); en esta investigación las mujeres utilizaron en menor proporción el condón, pero estas diferencias no fueron estadísticamente significativas.

La mayoría de las personas encuestadas no considera la posibilidad de que se puedan infectar con el VIH. Esto implica una baja percepción de riesgo y se refuerza por la falta de conocimientos completos acerca de esta enfermedad. Al ignorar o no tener la información adecuada, desconocen en consecuencia cómo adquirir la enfermedad y, más aún, cómo prevenirla; en un estudio realizado en Yucatán- México, sugieren que al parecer, las estrategias educativas utilizadas en las campañas no han sido suficientemente efectivas y no logran llegar a poblaciones de mayor vulnerabilidad (Pavía-Ruz, Góngora-Biachi, Vera-Gamboa, Moguel-Rodríguez y González-Martínez, 2012).

En una revisión donde se incluyeron estudios que comparaban las tasas de infección por VIH en grupos que aseguran siempre utilizar preservativo y aquellos que dicen nunca usarlo, se halló que el uso constante de preservativos da como resultado una reducción del $80 \%$ en la incidencia del VIH (Wilkinson, 2002); se requieren esfuerzos para mejorar los conocimientos y estrategias efectivas de prevención y que lleven a prácticas de sexo seguro y uso consistente del condón por parte de los usuarios de drogas inyectables.

El conocimiento sobre el VIH/Sida es casi universal, la mayoría de la población ha oído hablar de la enfermedad. Sin embargo, cuando se ahonda en el conocimiento integral de la misma, se encuentran vacíos que podrían incrementar la transmisión debido a las falencias frente a las medidas de prevención (Profamiilia, 2010); en la presente investigación el 93,3\% de la población había recibido información sobre el VIH, un 64,4\% informó que desde que escuchó hablar por primera vez de VIH/SIDA ha hecho algo para evitar infectarse o evitar infectar a otros, pero un 
$42 \%$ reportó haber compartido parte del equipo (agujas, mezclador, jeringa).

En un estudio en Costa Rica identificaron mayores conocimientos sobre prevención de VIH,al tener una relación de pareja en la actualidad y nunca haber consumido drogas (Vargas, 2006); en este estudio toda la población tenía un alto policonsumo de drogas, un 7,3\% de los UDI tenía una pareja sexual que también era UDI, y un $82 \%$ eran solteros; aspectos que se consideran factores de riesgo y presuponen una mayor vulnerabilidad en este grupo.

Como limitaciones de esta investigación se puede mencionar que el muestreo basado en el entrevistado es considerado un tipo de selección no aleatoria que debe cumplir una serie de supuestos para ser considerado una muestra dependiente de la red social, se reconoce que no se alcanzaron poblaciones de estratos socioeconómicos altos, los cuales no estarían interesados en recibir un incentivo por participar; además estos datos provienen de una encuesta que recolectó auto-reportes de prácticas sexuales y de uso de condón, pudiendo tener imprecisiones basadas en la honestidad de la respuesta, afectando el resultado final de ser un método efectivo de prevención (Grangeiro et al., 2012).

Los conocimientos sobre prevención de VIH y baja utilización del condón, demuestran una comprensión superficial, desinformación y por consiguiente un mayor riesgo de adquirir la infección por VIH. En un contexto epidemiológico donde el VIH se transmite principalmente por relaciones sexuales y en el cual hay presencia de infecciones de transmisión sexual, conocer y monitorear la falta de información, las concepciones erróneas y las conductas sexuales de esta población, son aspectos clave para diseñar intervenciones más efectivas.

Los conocimientos inadecuados sobre VIH y las conductas auto reportadas por los UDI, representan una alerta en las ciudades de Colombia, indicando la presencia de comportamientos de riesgo y poniendo de relieve la importancia de generar nuevas y más específicas intervenciones para la reducción de daños y la prevención del VIH en esta población.

\section{Reconocimientos}

Investigación financiada por el Ministerio de Salud y Protección Social de Colombia.

\section{Conflictos de interés}

No se tienen conflictos de interés.

\section{Referencias}

Abdul-Quader, A. S., Heckathorn, D. D., Sabin, K. y Saidel, T. (2006). Implementation and analysis of respondent driven sampling: lessons learned from the field. Journal of Urban Health, 83. doi:10.1007/s11524-006-9108-8.

Ball, A. L., Rana, S. y Dehne, K. L. (1998). HIV prevention among injecting drug users: responses in developing and transitional countries. Public health reports, 113, 170181.

Berbesi D, Montoya L. y Segura A. (2012). Prevalencia de VIH y comportamientos de riesgo en UDI en Medellin y Pereira. Bogota: Ministerio de Proteccion Social.

Bonell, C. y Imrie, J. (2001). Behavioural interventions to prevent HIV infection: rapid evolution, increasing rigour, moderate success. British medical bulletin, 58, 155170.

Bravo, M. J., Royuela, L., Barrio, G., Rodríguez-Arenas, M. A. y de la Fuente, L. (2004). [Prevalence of indirect sharing of drug-injecting paraphernalia in Galicia, Madrid, Seville and Valencia [Spain]. Gaceta sanitaria, 18, 472478.

Carnicer-Pont, D., Vives, N. y Casabona i Barbarà, J. (2011). Epidemiología de la infección por virus de la inmunodeficiencia humana. Retraso en el diagnóstico. Enfermedades Infecciosas y Microbiología Clínica, 29, 144-151. doi:10.1016/j.eimc.2010.11.010.

Chiao, C., Morisky, D. E., Ksobiech, K. y Malow, R. M. (2009). Promoting HIV testing and condom use among Filipina commercial sex workers: findings from a quasi-experimental intervention study. AIDS and behavior, 13, 892-901. doi:10.1007/s10461-008-9418-9.

Cobos Calleja, T. de los, Casanueva Gutiérrez, M. y Jove González, C. (2003). Perfil de los usuarios de drogas ingresados en un hospital. Anales de Medicina Interna, 20. doi:10.4321/S0212-71992003001000002.

Grangeiro, A., Holcman, M. M., Onaga, E. T., Alencar, H. D. R. de, Placco, A. L. N. y Teixeira, P. R. (2012). Prevalence and vulnerability of homeless people to HIV infection in São Paulo, Brazil. Revista de saude publica. Recuperado a partir de http://www.ncbi.nlm.nih.gov/ pubmed/22715004.

Hagan, H., Campbell, J. V., Thiede, H., Strathdee, S. A., Ouellet, L., Latka, M.,... Garfein, R. S. (2007). Injecting alone among young adult IDUs in five US cities: evidence of low rates of injection risk behavior. Drug and alcohol dependence, 91, S48-55. doi:10.1016/j.drugalcdep.2007.02.002

March, J. C., Oviedo-Joekes, E. y Romero, M. (2007). Inconsistent condom use among socially excluded heroin users. Gaceta sanitaria, 21, 321-328.

Mathers, B. M., Degenhardt, L., Ali, H., Wiessing, L., Hickman, M., Mattick, R. P.,... Strathdee, S. A. (2010). HIV prevention, treatment, and care services for people who inject drugs: a systematic review of global, regional, and 
national coverage. The Lancet, 375(9719), 1014-1028. doi:10.1016/S0140-6736(10)60232-2.

Mendez Diz, A. M., Schwarz, P. y Camarotti, A. C. (2009). Prácticas sexuales en usuarios de drogas y riesgo de transmisión del VIH/sida. Revista argentina sociología, 7 , 150-163.

Ministerio de la Proteccion Social. Republica de Colombia. (2008). Plan Nacional de respuesta ante el VIH y el SIDA. Colombia 2008 - 2011.

Ministerio de Salud y Proteccion Social. (2012). Informe mundial de avances en la lucha contra el Sida. Colombia 2012. Recuperado a partir de http://www.unaids.org/ en/dataanalysis/knowyourresponse/countryprogressreports/2012countries/.

Naciones Unidas. (2011). Declaración Politica sobre el VIH/ SIDA: intensificación de nuestro esfuerzo para eliminar el VIH/SIDA. Recuperado 9 de octubre de 2012, a partir de http://www.un.org/ga/search/view_doc.asp?symbol=A/65/L.77yreferer=/english $/$ yLang $=$ S.

NIDA. (2001). Principios para la Prevención del VIH en las Poblaciones de Usuarios de Drogas: Una guía basada en la investigación - Investigaciones. Recuperado 9 de octubre de 2012, a partir de http://archives.drugabuse.gov/ $\mathrm{POHP} / \mathrm{sp} /$ Investigaciones.html.

ONUSIDA. (2012). Datos fundamentales sobre el sida. Recuperado a partir de http://search2.unaids.org/custom/ search.asp.

Organización Mundial de la Salud. (2010). Factores relacionados al uso del condón en Paraguay Encuesta Nacional de Salud Sexual y Reproductiva 2004. Recuperado a partir de http:/ / www.cepep.org.py/archivos/Usocondonpy.pdf.

Organizacion Mundial de la Salud, y ONUSIDA. (2000). Guías sobre la vigilancia del VIH de segunda generacion. Recuperado a partir de http://cedoc.cies.edu.ni/ general/2nd_Generation \%20\%28D\% 29/Surveillan- ce \% 20Guidelines /2nd\%20Generation \% 20Surveillance/2nd\%20gen\%20Spa.pdf.

Pavía-Ruz, N., Góngora-Biachi, R., Vera-Gamboa, L., Moguel-Rodríguez, W. y González-Martínez, P. (2012). Conocimientos, actitudes y percepción de riesgo en referencia al VIH/SIDA en población rural de Yucatán, México. Revista Biomédica, 23, 53-60.

Prevention of acquired immune deficiency syndrome (AIDS): report of inter-agency recommendations. (1983). MMWR. Morbidity and mortality weekly report, 32, 101-103.

Profamiilia. (2010). Conocimiento del VIH entre las mujeres y su percepción del riesgo: Encuesta Nacional de Demografia y Salud Colombia. Recuperado a partir de http://www.profamilia.org.co/encuestas/.

Shisana, O., Rice, K., Zungu, N. y Zuma, K. (2010). Gender and poverty in South Africa in the era of HIV/AIDS: a quantitative study. Journal of women's health, 19, 39-46. doi:10.1089/jwh.2008.1200.

Uribe, J. I., Amador, G., Zacarias, X. y Virrareal, L. (2012). Percepciones sobre el uso del condón y la sexualidad entre jóvenes. Revista Latinoamericana de Ciencias Sociales, Niñez y Juventud, 10, 481-494.

USAID. (2010). Conocimiento de VIH/SIDA, Otras ETS y Formas de Prevención. Recuperado a partir de http://www. measuredhs.com/.

Vargas, R. (2006). Conocimientos, actitudes y prácticas VIH/ sida y uso del condón. Recuperado a partir de http:/ / www. cipacdh.org/pdf/VIH\% 20SIDA\% 20CONDON\% 20 TRAILEROS\%20CIPAC.pdf.

Wilkinson D. (2002). Efectividad del preservativo en la reducción de la transmisión del VIH en heterosexuales: Comentario de la BSR. Organización Mundial de la Salud. Recuperado a partir de http://apps.who.int/rhl/hiv_aids/dwcom/es/index.html. 